\title{
Humanizing the Chart: Becoming More Responsive to Patient Needs Through Implementation of PatientWisdom
}

\author{
Bradley Crotty ${ }^{1}$, MD, MPH; Jeana Holt ${ }^{2}$, DNP, RN; Rachel Cusatis ${ }^{2}, \mathrm{PhD}$; Onur Asan ${ }^{3}, \mathrm{PhD}$; Joni Williams ${ }^{3}, \mathrm{MD}_{\text {, }}$ \\ MPH; Kathryn Flynn ${ }^{4}$, PhD; Jennifer Moore ${ }^{5}$, MHA; Gregory Makoul ${ }^{5}$, PhD \\ ${ }^{1}$ Collaborative for Healthcare Delivery Science, School of Medicine, Froedtert \& Medical College of Wisconsin, Milwaukee, WI, United States \\ ${ }^{2}$ Academic Fellowship in Primary Care, Medical College of Wisconsin, Milwaukee, WI, United States \\ ${ }^{3}$ Center for Advancing Population Science, Medical College of Wisconsin, Milwaukee, WI, United States \\ ${ }^{4}$ Center For International Blood \& Marrow Transplant Research, Medical College of Wisconsin, Milwaukee, WI, United States \\ ${ }^{5}$ PatientWisdom, New Haven, CT, United States
}

\section{Corresponding Author:}

Bradley Crotty, MD, MPH

Collaborative for Healthcare Delivery Science

School of Medicine

Froedtert \& Medical College of Wisconsin

8701 Watertown Plank

Milwaukee, WI, 53226

United States

Phone: 15323783

Email: bcrotty@mcw.edu

\begin{abstract}
Background: Over the past several decades, health care has been shifting to a care model that more fully values patient engagement. Recently, there has been increased attention on the role of health information technology that enables patients to collaborate with clinicians through the sharing of patient-generated contextual data. We implemented the PatientWisdom tool using a sociotechnical model to improve patient experience and visit effectiveness.

Objective: To understand the facilitators and barriers to the routine incorporation of patient contextual data into the record, and the subsequent initial impact on the experience of care within academic and community practices affiliated with an academic health system.

Methods: Our health system co-developed the PatientWisdom tool, which elicits patient values, preferences, and other contextual data ahead of visits through an email invitation to a secure Web application. Results are summarized and viewable within the EHR. To assess the implementation, we performed workflow shadowing and semi-structured interviews of clinical staff from April through July 2018. The Consolidated Framework for Implementation Research (CFIR) guided the collection and analysis of qualitative data. Researchers used the PatientWisdom platform to elicit patient data ahead of visits and summarize insights in the EHR. The researchers conducted sampling and data analysis in tandem; sampling concluded when the researchers determined no new themes were surfacing; therefore reaching data saturation. To assess the impact of the program on health care operations, including patient experience, a random sample of clinicians and patients (both users and non-users) was performed.

Results: In workflow assessment and clinician interviews, we learned that the data needed to be more visible within the chart, and we made improvements within our electronic health record to make link to the data more apparent and visible when data were available. Main themes from our interviews were: Patient contextual information fosters a holistic approach to care; PatientWisdom is an innovative tool used to sync clinician-patient goals; clinicians may have an incorrect perception of PatientWisdom adopters (expecting millennials); and clinicians who proactively integrate PatientWisdom into their workflow identified it as an asset to care. In our initial assessment of impact, 945 patients completed surveys following their visits. Of participating patients, $87 \%$ say it improves communication and $90 \%$ rate the visit as going "extremely well" (compared to $82 \%$ when not used $P<.05)$. Clinicians were significantly more likely to ask patients about barriers to care using the tool $(65 \%$ vs $48 \%)$. The tool surfaced information about patient needs for clinicians and leaders. For example, we identified that $47 \%$ of participants did not have a health care proxy, and $60 \%$ of these patients were ready to talk about it with their clinician.
\end{abstract}


Conclusions: Consumer informatics tools that link with electronic health records may help "humanize" the record and improve patient centeredness. This session highlights the initial implementation of an effort to collect patient-contextual data through the PatientWisdom tool and highlights the use of the tool to drive clinical and operational improvement.

(iproc 2018;4(2):e11793) doi: $\underline{10.2196 / 11793}$

\section{KEYWORDS}

communication; consumer health informatics; electronic health record; implementation; sociotechnical

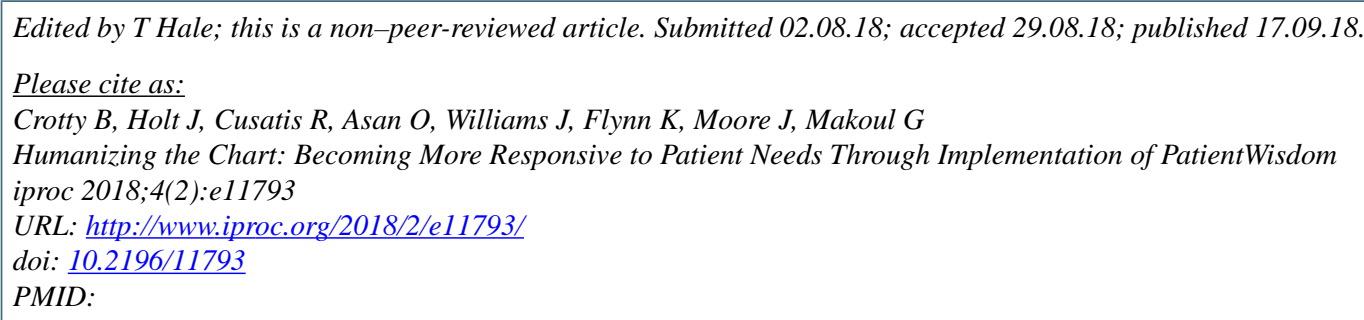

CBradley Crotty, Jeana Holt, Rachel Cusatis, Onur Asan, Joni Williams, Kathryn Flynn, Jennifer Moore, Gregory Makoul. Originally published in Iproceedings (http://www.iproc.org), 17.09.2018. This is an open-access article distributed under the terms of the Creative Commons Attribution License (https://creativecommons.org/licenses/by/4.0/), which permits unrestricted use, distribution, and reproduction in any medium, provided the original work, first published in Iproceedings, is properly cited. The complete bibliographic information, a link to the original publication on http://www.iproc.org/, as well as this copyright and license information must be included. 